\title{
ESTRATEGIAS DE APRENDIZAJE UTILIZADAS POR ALUMNOS DE PRIMER AÑO DE UN CURSO DE FORMACIÓN DE PROFESORES DE EDUCACIÓN MEDIA
}

Inés Rivero*

\section{Propósito}

En este especial momento de la evolución cultural humana es indiscutible que el principal papel que le corresponde a la educación en tanto herramienta que permita la constante reconstrucción y creación de cultura, es el de brindar oportunidades a los alumnos a fin de que éstos desarrollen habilidades para continuar aprendiendo en forma autónoma: es el concepto de "aprender a aprender», presente en numerosa bibliografía de uso cotidiano para los docentes. Esta constituye sin duda una valiosa capacidad abierta, cuya adquisición posibilita a su vez continuar aprendiendo durante toda la vida, en la medida en que cada individuo cree y guíe su propio aprendizaje

Uno de los mecanismos para lograr este objetivo es la utilización de procedimientos particulares, elegidos por cada estudiante de acuerdo con las exigencias y las características de la situación específica. Estos procedimientos son el producto de un largo proceso de construcción personal que se lleva a cabo durante toda la vida. Existe consenso entre los autores (Marchesi y Martín, 1998; Pozo,1999; Monereo, 1998; Resnick y Klopfer, 1996) con respecto a que es indispensable que estos mecanismos sean enseñados en forma intencional. En nuestro sistema educativo, sin embargo, son escasas las oportunidades que se brindan a los estudiantes para que puedan construir formas de actuar que les permitan hacer más efectivo su aprendizaje. Tanto a nivel de Educación Primaria como de Educación Secundaria, el énfasis se encuentra en los contenidos curriculares más que en los procedimientos eficaces para aprehenderlos. Los estudiantes, en todo caso lograrán diseñar en forma independiente y autónoma sus propios guiones de acción.

Resulta interesante entonces indagar en qué consiste la batería de recursos facilitadores que logran diseñar los estudiantes a lo largo de su escolaridad, sin una instrucción específica. Para ello tomaré un grupo de estudiantes que ya cursaron con éxito doce años de enseñanza en el sistema educativo formal. Desarrollaré este estudio con alumnos de primer año de un instituto de formación de Profesores de Educación Media. Considero de especial importancia la elección específica de esta población, ya que estos jóvenes han elegido la docencia como carrera; la necesidad de construir mecanismos de aprendizaje es más relevante aun para ellos, pues deberán mantenerse actualizados a partir de una formación inicial que no cubre las demandas del ejercicio profesional. El egresado de formación docente necesita de los primeros años de ejercicio profesional para configurar un cuerpo de conocimientos que le permita desenvolverse con solvencia en el aula. A esto se agrega el constante cambio que sufre el rol del docente, y que ha dado sobrados motivos para plantear el tema de la formación permanente del profesorado: Paralelamente, en el desempeño de su práctica profesional, estarán obligados a ser capaces de crear instancias para que sus alumnos desarrollen este tipo de habilidades. Por este motivo, indagaré cuáles son los procedimientos de aprendizaje que manifiestan utilizar, en forma consciente, intencional y planificada, los estudiantes de primer año de un centro de formación de profesores de Educación Media. Del resultado de esta investigación espero obtener información que permita establecer, para los alumnos consultados, si en el transcurso de su escolaridad y sin una enseñanza especial, han desarrollado mecanismos que les faciliten el abordaje del conocimiento y cuáles son los más frecuentemente utilizados. 


\section{Marco teórico}

Entre los muchos desafíos que enfrenta la educación en la actualidad, el principal es, sin duda, la transformación que ha sufrido el papel de los educadores. Ya no son transmisores de saberes completos o "acabados". En los últimos años, los docentes han sido, entre otras: animadores, orientadores, promotores, facilitadores. Detrás de esta infinidad de caracterizaciones subyacen los mismos problemas: el papel activo que corresponde a cada sujeto en su aprendizaje y la individualidad de dicho proceso en lo que se refiere al plano personal y paralelamente, la constante multiplicación de los contextos de aprendizaje y sus metas en el plano cultural. Se verifica un cambio tanto en lo que se aprende como en la forma de aprenderlo. Ya no basta con dominar contenidos fácticos. Los saberes están sujetos al fenómeno que Chevallard ha denominado "desgaste"; es decir, pérdida de vigencia debido a nuevos saberes.

Resulta imprescindible entonces conocer los procedimientos que permitan mantener el caudal de conocimientos actualizado. Así, los estudiantes obtendrán más provecho del tiempo que invierten en aprender si lo hacen con un abanico de recursos de tipo procedimental entre los que puedan elegir libremente y utilizar en forma estratégica, esto es, seleccionándolos de acuerdo con las exigencias de cada tarea. Es particularmente importante que los docentes sean conscientes de las estrategias que utilizan; la formación inicial no cubre las demandas del ejercicio profesional. Al respecto, es posible citar a Torres (1996: 55). "La necesidad de una reforma profunda del modelo escolar y del currículo tradicional de la educación básica está hoy instalada en la mayoría de países en desarrollo y viene cobrando forma en muchos de ellos"... "La necesidad de revisar el rol convencional de los maestros surge como corolario de la realidad educativa actual y de los modernos planteamientos y procesos de cambio. El ideal docente hoy no es el maestro-instructor y el maestro enciclopedia del pasado, sino el docente que percibe su rol como provocador y facilitador de aprendizajes y asume su misión no en términos de enseñar sino más bien de lograr que los alumnos aprendan; el profesional que está en capacidad no sólo de interpretar y aplicar un currículo, sino de recrearlo y construirlo él mismo" y a Gimeno Sacristán: La formación inicial del profesorado opera con la creencia implícita de que puede producir competencias profesionales que después se pondrán en acción cuando los nuevos profesores se incorporen a su práctica profesional. Tal presupuesto se liga a una creencia implícita optimista sobre la fecundidad del conocimiento pedagógico y de la experiencia de formación inicial para la práctica de la educación, lo que la mayoría de las veces es un a priori no demostrado. Los estudios sobre la socialización de los profesores destacan con regularidad que la preparación de profesores es una tarea normalmente de "bajo impacto" en la configuración de la profesionalidad y que sus defectos son débiles (Zeichner, U. 1981)" Gimeno Sacristán, José ( 1998: 128).

Por lo anteriormente expuesto, el docente, para desenvolverse adecuadamente, necesita mantener constantemente una actitud abierta y crítica a la cultura y la disposición para aprender en forma autónoma durante toda la vida. Ello es especialmente válido para estos estudiantes, ya que se ven involucrados en una experiencia innovadora, que aún está sufriendo ajustes importantes. Como lo afirman Marchesi y Martín (1998:157): "Los primeros años de los profesores, sobre todo para aquellos sin experiencia previa o que se encuentran con grupos de alumnos muy heterogéneos o muy problemáticos, son muy difíciles. Los profesores no han constatado en la práctica cuáles son las estrategias más adecuadas para conseguir que su grupo de alumnos alcance los objetivos educativos planteados".

Estos futuros docentes deberán hacerse cargo de un grupo de clase, como una instancia obligatoria del tercer año de su carrera, luego de solo dos años de formación teórica, en la que tuvieron escasamente un mes de práctica de aula. Se espera que construyan un caudal significativo de aprendizajes a medida que se desenvuelvan como docentes, razón por la cual necesitarán ser sumamente eficientes en su desempeño. Se agrega a esto el hecho de que la mayoría proviene de hogares con poco capital cultural relativo, información que se desprende de las fichas individuales que los alumnos completan al solicitar la inscripción; en un alto porcentaje de las familias, sus integrantes no han completado la Educación Secundaria, siendo ellos los representantes del mayor nivel educativo alcanzado en su núcleo familiar. 
La preparación de las clases les insumirá posiblemente un tiempo considerable, ya que son importantes los contenidos que deben dominar; no solo los relativos al logro de la textualización de los saberes, sino también a los saberes específicos a tratar. Desde otra óptica, y por la carrera que han elegido, estos alumnos deberán generar en sus aulas oportunidades para que quienes estén a su cargo configuren a su vez sus propias estrategias.

Ejercerán la docencia en el ciclo medio de enseñanza, enfrentándose con grupos de alumnos para quienes este ciclo es el final de la educación formal. Será esta, para muchos de ellos, la última oportunidad de recibir instrucción y de elaborar mecanismos que les posibiliten continuar aprendiendo en forma independiente. De allí la importancia de que sus docentes dominen los procedimientos que facilitan el aprender, para que a su vez sean capaces de enseñárselos.

En el diseño de este trabajo he tomado el concepto de aprendizaje como un proceso de organización cognitiva constante: "Saber algo no es sencillamente haber recibido la información sino también haberla interpretado y relacionado con otros conocimientos. Ser experto no es solo saber cómo desempeñar una acción sino también saber cuándo desempeñarla y adaptar el desempeño a las diversas circunstancias". (Resnick y Klopfer 1989:19), en tanto que he utilizado la siguiente definición de estrategias de aprendizaje: "podemos definir las estrategias de aprendizaje como procesos de toma de decisiones (conscientes e intencionales) en los cuales el alumno elige y recupera, de manera coordinada, los conocimientos que necesita para cumplimentar una determinada demanda u objetivo, dependiendo de las características de la situación educativa en que se produce la acción". (Monereo 1998:27). Se refiere a los conocimientos que deben ir desarrollando los alumnos a lo largo de su escolaridad de manera que puedan enfrentarse a nuevos aprendizajes con éxito. Estas habilidades se consideran actualmente uno de los principales objetivos de la educación: capacitar a los estudiantes para "aprender a aprender".

En la noción de estrategia de aprendizaje resulta determinante la intencionalidad y el control que ejerce el sujeto en todo el proceso: "Un uso estratégico se caracterizaría porque el alumno es capaz de controlar de forma deliberada e intencional la ejecución y, sobre todo, porque puede seleccionar el procedimiento que en cada caso resulta más adecuado, mediante un análisis de las características específicas de la tarea"... "No se trata simplemente de enseñar a los alumnos a organizar la información o a recordarla eficazmente a través de determinados recursos mnemotécnicos, sino de instruirles en la regulación selectiva y controlada de esas estrategias (Monereo y Castelló, 1997)". (Marchesi y Martín, 1998: 363).

De acuerdo con estos supuestos teóricos, el acto de aprender asume ciertas características particulares, propias de la concepción constructivista del aprendizaje: el aprender es un proceso intencional por parte del sujeto, donde están involucrados no solamente sus capacidades sino también sus motivos e intereses. Implica regulación y control durante el desarrollo de la actividad y el conocimiento del propio funcionamiento intelectual: qué sabe, cómo aprende mejor, de qué manera puede utilizar sus conocimientos. Un nivel de pensamiento de este tipo supone haber desarrollado capacidades metacognitivas. El aprendizaje está entonces ligado al concepto de metacognición, entendiendo ésta como el conocimiento del propio funcionamiento intelectual. De acuerdo con los psicólogos evolutivos, la metacognición forma parte del "conocimiento de sí mismo" o "autoconcepto" (Palacios, Marchesi, 1990:278 y 279) y su desarrollo se inicia hacia los 7 u 8 años de edad, alcanzando su máxima expresión en la adolescencia. A partir de este período el sujeto se define a sí mismo con atributos de índole psicológica más que física. Puedo suponer entonces, que jóvenes que tienen ya más de 18 años y han finalizado el ciclo secundario, con el papel que indiscutiblemente tiene la educación en la evolución intelectual, hayan desarrollado completamente el conocimiento de su propio yo. No tendrán dificultades, entonces, en analizar cuáles son los procedimientos que ponen en juego cuando necesitan aprender

Para afinar más la concepción de estrategias de aprendizaje debemos tener en cuenta que está comprometida también la actitud del alumno frente a la tarea. Pozo distingue entre lo que denomina "enfoque superficial" y "enfoque profundo". En el primero de los casos, el aprendiz entiende el conocimiento como acumulación de contenidos. Tiende a reproducir los saberes. Su expresión característica 
corresponde al aprendizaje memorístico de Ausubel. En el "enfoque profundo", se busca la comprensión y la integración del nuevo saber a los ya adquiridos. Se identifica con el aprendizaje significativo. El alumno tiene una actitud mucho más activa. Se interroga a sí mismo y al nuevo material a fin de comprenderlo cabalmente; pone entonces en juego diferentes estrategias. Debido a que en el enfoque superficial se busca el recuerdo más o menos literal de la información, predomina la memorización y el repaso como técnica de apoyo. Cuando un alumno está verdaderamente comprometido con la tarea, en cambio, establecerá relaciones entre los materiales nuevos y los ya conocidos, utilizando o no diferentes representaciones gráficas, logrando la reorganización y reestructuración del saber.

No está en discusión actualmente la necesidad de enseñar a utilizar estrategias de aprendizaje. Sin embargo, no hay acuerdo entre los autores en realizar una instrucción independiente de los contenidos o que ella esté íntimamente ligada a los mismos. Personalmente, considero que el aprendizaje, y por lo tanto la enseñanza, deben abordarse integrados al saber a enseñar o a aprender. Sí entiendo deseable que cada procedimiento de aprendizaje se trabaje con contenidos disímiles, de forma de facilitar luego la integración de los mismos en estrategias que resulten efectivas en diferentes áreas del conocimiento.

Como resultado de esta investigación, y de acuerdo con el marco teórico seleccionado, espero poder establecer cuáles son las estrategias de aprendizaje que describen algunos alumnos que inician la educación en el nivel terciario. Esto será posible si efectivamente los sujetos a investigar han desarrollado la metacognición lo suficiente como para poder relatar los procesos mentales a los que recurren habitualmente. En caso de que no fueran capaces de realizarlo, podría pensar que no han desarrollado estrategias de aprendizaje en el sentido estricto del término, ya que una de las condiciones sine qua non de las mismas es la aplicación intencional y la autorregulación cognitiva constante. En tal situación solamente podría hablar de utilización de procedimientos para aprender, pero no de estrategias.

\section{Metodología}

\section{Pregunta de investigación}

¿Cuáles son las estrategias de aprendizaje que dicen utilizar los estudiantes de primer año de un centro de formación de profesores de Educación Media?

He planteado esta indagación dentro del paradigma cualitativo, buscando interpretar los mecanismos de acción mental particulares de cada entrevistado, atendiendo al concepto de estrategia ya suficientemente explicitado. Para desarrollar este trabajo recurrí a entrevistas semiestructuradas, entendiendo éstas como ..."encuentros cara a cara entre el investigador y los informantes, encuentros éstos dirigidos hacia la comprensión de las perspectivas que tienen los informantes respecto de sus vidas, experiencias o situaciones, tal como las expresan con sus propias palabras". (Taylor y Bogdan, 1984:101):

Claramente, por tratarse de procesos de índole psicológica, totalmente internos al sujeto, considero que ésta es la técnica más apropiada para indagar cuáles son las estrategias de aprendizaje utilizadas por adultos jóvenes, pues se relaciona con tareas cotidianas en la vida del estudiante. Este es el criterio de validez inferencial o sistémica. Con el objetivo de obtener la información necesaria, construí una pauta que utilicé como guía y fui introduciendo preguntas adicionales a medida que la situación lo requería. Se puede decir que el muestreo fue de tipo intencional. Para seleccionar el grupo a entrevistar, comenté que necesitaba entrevistar a algunos estudiantes para una tarea que debía presentar y trabajé con los que se ofrecieron voluntariamente. Me parece importante destacar esta situación, pues en esta indagación es fundamental la colaboración del sujeto, que fácilmente podría brindar información falsa o inexacta si se viera obligado a responder. Desarrollé este estudio en un instituto de formación docente, en el que me desempeño como profesora, y con los alumnos de primer año, ya que recién encaran su formación profesional y por lo tanto no han tenido oportunidad de desarrollar nuevas estrategias de aprendizaje desde que egresaron de Educación Secundaria. 
Siguiendo las ideas presentes en los autores consultados, estos jóvenes resultan expertos en el aprendizaje, especialmente en las áreas que han elegido para especializarse: Lengua y Literatura, Ciencias Sociales, Matemática. Un sujeto experto es aquél que ha desarrollado un caudal de conocimientos y de procedimientos que puede poner en juego a voluntad para hacer más eficaz su aprendizaje. Sin embargo, posiblemente actúen como novatos en el abordaje de las asignaturas de Ciencias de la Educación, las que en muchos casos requieren un tratamiento diferente al que pueden haber desarrollado hasta el momento. Es de esperar que dada su experiencia en actividades de aprendizaje se comporten como "novatos inteligentes", es decir, que sean capaces de utilizar estrategias ya dominadas para otras áreas del saber en el área de Ciencias de la Educación.

A pesar de que cada entrevista siguió la misma pauta general, fui introduciendo preguntas que ampliaran información -si ello era necesario-, o sobre aspectos que abrieran nuevas visiones del mismo fenómeno. Trabajé con los alumnos que se ofrecieron voluntariamente de los grupos que cursan profesorado en las áreas de: Lengua y Literatura, Ciencias Sociales y Matemática. Me interesó particularmente tomar sujetos de diferentes áreas de profesorado para analizar cómo enfrentan el aprendizaje alumnos que prefieren tan diferentes enfoques del conocimiento. A fin de mantener la relación con la población total, entrevisté en forma proporcional a la cantidad de alumnos inscriptos en cada curso, hasta un total de 17 casos.

\begin{tabular}{|lccc|}
\hline Área & $\begin{array}{l}\text { Número } \\
\text { de alumnos }\end{array}$ & Entrevistados & $\begin{array}{l}\text { \% en relación } \\
\text { a la cantidad de alumnos }\end{array}$ \\
\hline Matemática & 12 & 5 & $42 \%$ \\
\hline Ciencias Sociales & 18 & 7 & $43 \%$ \\
\hline Lengua y Literatura & 12 & 5 & $42 \%$ \\
\hline
\end{tabular}

Durante la consulta, que realicé en forma individual y privada a cada uno de los sujetos, les solicité que describieran los procedimientos que acostumbran utilizar cuando debe enfrentarse a una situación de aprendizaje "en solitario". Dicha técnica supone que los entrevistados han desarrollado tanto la metacognición como las estrategias de aprendizaje a un nivel suficiente como para reconocerlas y describirlas. Este procedimiento está de acuerdo con la investigación, ya que la autorregulación es una de las características de la utilización de las estrategias de aprendizaje. Este es, sin duda, un factor limitante en la investigación: si los entrevistados no pueden describir a solicitud los procedimientos que utilizan para aprender, esta consulta no sería válida. Sin embargo, entiendo que es menos riesgoso que someter a los sujetos a situaciones de aprendizaje para luego evaluar su desempeño. A pesar de ser docente de estos alumnos, no considero posible analizar procesos que son de índole interna a través de su desempeño en el aula. Entiendo que no cuento con los instrumentos que me permitan la observación directa de la utilización de estrategias específicas, y carezco de la experiencia indispensable para interpretar procesos mentales a través de acciones concretas. 


\section{Análisis}

En las entrevistas identifiqué diferentes categorías, dado que en las respuestas de los sujetos es posible reconocer estrategias de diversa índole:

estrategias de reorganización (de acuerdo con Selmes: enfoque profundo):

- Busca una interpretación personal.

- Contrasta sus idea sobre el tema con las de otras personas/autores.

- Vincula el tema con su experiencia previa.

- Aplica lo aprendido en situaciones particulares/ejercicios.

- Vincula el tema con otros temas.

- Establece relaciones de jerarquía entre segmentos del material.

- Busca información complementaria.

- Utiliza estrategias de reorganización para el repaso.

El aprendiz que encara su aprendizaje con un enfoque profundo analiza activamente la estructura del material, identifica las interrelaciones entre el mismo y los conocimientos previos, busca integrar el nuevo saber a su estructura cognitiva. El resultado de este esfuerzo es la oportunidad de ampliar las posibilidades de aplicación de dicho conocimiento en contextos variados y diferentes, así como la adopción de un punto de vista personal.

estrategias de elaboración (enfoque superficial):

- Predomina la memorización

- Utiliza segmentos textuales del material.

- Utiliza estrategias de reproducción para el repaso.

Son procedimientos que utiliza el estudiante para interpretar un material. Establece relaciones entre segmentos de un mismo texto o, en un nivel de mayor complejidad, con materiales diferentes..No se da aún la reorganización del conocimiento, si bien éste puede reproducirse. El alumno adquiere el conocimiento pero no puede utilizarlo a voluntad en situaciones variadas. El saber adquiere la característica que Perkins ha denominado "conocimiento inerte".

\section{estrategias de planificación y estrategias metacognitivas.}

- Trabaja mejor solo.

- Trabaja mejor en equipo.

- Varía las estrategias de acuerdo con su interés.

- Varía el tipo de procedimiento de acuerdo al contenido/dificultad.

- Planifica el lugar/tiempo de estudio.

- Controla su aprendizaje mientras aprende.

- Controla su aprendizaje al final.

- Explica su forma de proceder con dificultad.

- Explica su forma de proceder sin dificultad. 
Aquí se refleja el conocimiento de sí mismo y de las condiciones en que su aprendizaje es más eficaz, así como los recursos de que dispone cuando el material no le resulta interesante o accesible. Si bien se trata de ítemes diferentes, considero indispensable que las respuestas de cada entrevistado sean analizadas en su totalidad y de ese modo tener una idea del perfil que presenta como aprendiz.

En el caso de búsqueda de información complementaria y establecimiento de jerarquías entre el material, algunos alumnos lo realizan dentro de un enfoque profundo, acompanándolas de otras estrategias de reorganización. Sin embargo, también las utilizan estudiantes que se valen de la elaboración para aprender y en estos casos apoyan a la memorización Por este motivo, estas columnas han sido incluidas en el cuadro de estrategias de reorganización y en el de estrategias de elaboración, dejando los casilleros en negro cuando al analizar la respuesta es evidente que corresponde a un enfoque diferente.

A partir de estos cuadros y de las entrevistas, es posible obtener algunas conclusiones:

- Predomina el tipo de aprendizaje que Ausubel denomina de elaboración compleja (Coll, Palacios, Marchesi: 1988:209), que se encuentra a medio camino entre la asociación y la reorganización: si bien existe una cierta integración del conocimiento, éste permanece aislado en la estructura cognitiva del sujeto. No puede ser relacionado a voluntad con otros saberes. Correspondería al tipo de conocimiento que Perkins caracteriza como "conocimiento inerte" (Perkins; 1995). El sujeto lo posee, pero no puede utilizarlo a voluntad. Si bien se observan algunos procedimientos que corresponden al enfoque profundo, (aplicar lo aprendido en situaciones particulares, establecer relaciones de jerarquía entre el material),estos no se ven respaldados por otros recursos (contrastar sus ideas con el de otras personas/autores, búsqueda de una interpretación personal, utilización de estrategias de reorganización para el repaso) que les permitan lograr la integración conceptual.

- En general, han desarrollado un nivel de metacognición aceptable para jóvenes en el final de la adolescencia: saben en qué condiciones su desempeño es mejor.

- La mayoría reclama espacios para estudiar en solitario, sin interrupciones y, paralelamente, indican que el funcionamiento en equipo no les resulta provechoso: "En equipo trabajo bien cuando me tocan personas que se interesan y que trabajan. Son los riesgos del equipo: pero cuando trabajo sola yo, en el momento que puedo, lo puedo aprovechar. No tengo que estar coordinando, reunirme"... (E: $\left.\mathrm{N}^{\circ} 17\right)$ Cabe preguntarse entonces si existe en estos casos un andamiaje real entre pares, ya que excepto en tres casos, todos los demás manifiestan lograr mejor concentración cuando están solos. Un punto que no es posible soslayar a este respecto es el hecho de que casi la mitad de los estudiantes de este instituto poseen beca total, que incluye el alojamiento, durante la semana, en un hotel de la localidad. En ese lugar comparten una habitación entre 4 ó 6 estudiantes. Poseen algunos espacios que pueden utilizar como aulas para estudio, pero de ninguna manera existe la posibilidad física de que logren el aislamiento que reclaman. ¿Sería de esperar entonces que su rendimiento se viera afectado por estas condiciones?

- Todas las respuestas de los estudiantes, al consultarles cómo hacían para aprender, se refirieron a la utilización de material bibliográfico, ya sea textos, fotocopias, artículos de internet o apuntes de clase. Ninguno se planteó la posibilidad de aprender a partir de obras musicales, teatrales o pictóricas, material publicitario, programas de divulgación cultural, etc. Es decir que, aparentemente, no consideran factible que puedan adquirir conocimientos a través de otras manifestaciones de la cultura. Esto es particularmente grave para los alumnos de las áreas de Lengua y Literatura y Ciencias Sociales, pues justamente mucho de lo que habrán de transmitir deberá partir de su interpretación de la realidad cultural en que estén insertos. Desde otro ángulo, está muy difundido el papel de las diversas formas de inteligencia en la conformación psíquica de los sujetos. ¿Será posible que en estos sujetos estén tanto más desarrolladas las habilidades verbales y lógico-matemática que las otras? Si ello es así, indudablemente sería conveniente encontrar caminos para que puedan afianzar las que en apariencias tienen menor grado de manifestación. ¿Qué implicación tendrá esto para su futuro desempeño como docentes? ¿Podrán reconocer y brindar oportunidades de desarrollo a las diferentes capacidades de los alumnos a su cargo? 
- ¿Estos jóvenes eligieron la carrera por vocación? ¿Es el aprendizaje para ellos una instancia de crecimiento personal? Si así fuera en un número importante de casos, entonces pienso que deberían ser más frecuentes las respuestas afirmativas en cuanto a búsqueda de material complementario y la aplicación de lo aprendido a situaciones particulares, es decir, aquellas estrategias que revelan involucramiento con su formación y que estarían en condiciones de llevar a cabo aun cuando no hayan dominado los procedimientos de reorganización del conocimiento. Sin embargo, éstas se dan en contados casos. La impresión que tengo a partir de esta indagación es que los alumnos estudian porque tienen que estudiar, sin que exista un mayor compromiso de su parte. Su actitud está más cerca del estudiante de Educación Secundaria que de un alumno de Formación Profesional..

- Quienes más tienden a aplicar los conocimientos a situaciones particulares son los del área de Matemática. Es posible que esto se deba al estilo particular en que se aprende dicha materia: los alumnos estudian conocimientos teóricos e inmediatamente los aplican en ejercicios. El comportamiento de estos estudiantes en las otras áreas del conocimiento es el que se denomina de "novato inteligente" es decir, aplican estrategias que les han dado resultado para un tipo de saber específico, en otros que les son desconocidos. Cabría preguntarse si siempre podrán actuar de esta forma y si este comportamiento no actuaría finalmente como una limitante para el desarrollo de otros procedimientos diferentes, tal vez más apropiados para otros contenidos. Un interrogante más es si los alumnos de otras áreas se verían beneficiados del entrenamiento que han recibido estos jóvenes.

- Carecen también de recursos auxiliares cuando un material les resulta demasiado complejo. No recurren a otros materiales ni se valen de herramientas diferentes. El profesor es visto como el único recurso: "ahí sí voy y consulto" además de la revisión del tema a tratar. (E: $\left.\mathrm{N}^{\circ} 14\right)$

- Para las asignaturas que implican "lectura", la estrategia favorita, casi la única, es el subrayado. "Para recordar el tema creo que no sé, a mi será porque estaba acostumbrada a los esquemas que me resulten más fáciles que el mapa conceptual, que son medio complicados, si pasa un tiempo y no te acordás muy bien"... (E.N: 12). Los mapas conceptuales los realizan si el profesor lo solicita (solo un estudiante, el $\mathrm{N}^{\circ} 7$, afirma obtener mayor provecho de éstos que del subrayado). La dificultad más aludida es la de identificar los conectores, es decir, la vinculación y las relaciones de jerarquía entre los conceptos involucrados. Esto revela que el conocimiento no ha adquirido la reorganización que requiere para considerarse un saber integrado. Para muchos el indicador de logro del aprendizaje es la posibilidad de reproducir el saber utilizando vocabulario propio, más o menos exacto: "Cuando hago ese proceso.... no sé qué hago (se rié) pero cuando yo por ejemplo estudio de noche y en el día la cabeza está en eso y veo que no soy capaz de explicarlo yo con mis palabras y entenderlo yo diferente o igual a lo que dice el texto, ahi es cuando vuelvo... vuelvo y lo leo hasta comprenderlo yo"... (E:N: 13)

- Analizando su forma de proceder, no podría asegurar que hayan desarrollado una batería interesante de estrategias de aprendizaje. Dos alumnos (E. $\mathrm{N}^{\mathrm{o}}: 7$ y $\mathrm{N}^{\mathrm{o}}$ 14) ni siquiera se plantean la posibilidad, pues manifiestan que con una lectura les es suficiente. Los otros recurren al subrayado de la idea principal y a partir de allí elaboran o reorganizan el conocimiento. Me llama la atención que siempre procedan de la misma forma, lo que me hace pensar que no hay una intencionalidad manifiesta. En ese caso, estaríamos en presencia de procedimientos para aprender (utilización de un único procedimiento) pero de ningún modo sería posible hablar de estrategias de aprendizaje, de acuerdo con la definición de la que partí en este trabajo. 


\section{Discusión}

La intención de esta indagación era reconocer baterías de estrategias de aprendizaje desarrolladas por alumnos que ingresan al nivel terciario. No puedo afirmar que haya encontrado dichas baterías. Esperaba encontrar al menos dos o tres procedimientos en cada uno. En cambio, el recurso utilizado por la casi totalidad se reduce al subrayado. Algunos, a partir de esa selección, elaboran un esquema con una cierta organización, pero este esquema cobra aparentemente, el carácter de trabajo final. Con su elaboración se da por finalizada la sesión de estudio. No se lo utiliza como soporte para reorganizar el saber.

Tampoco observo la planificación de acciones dirigidas al logro del conocimiento, a pesar de que ellos saben cuáles son las condiciones que requieren. Los alumnos asumen que deben estudiar y en general lo hacen frente a una instancia de evaluación, sin establecer, además de una secuencia de acciones, un cronograma que les permita administrar su tiempo, que debido al que les insumen los desplazamientos a y desde el instituto, se ve bastante reducido. Reflejo de ello es la dificultad que encuentran cuando deben funcionar en equipo. Es posible sí identificar una cierta intencionalidad de aprender, de acuerdo con el concepto que cada uno tiene de qué significa aprender, pero parecen carecer totalmente de los recursos indispensables para que ese aprendizaje tenga la eficacia a esperar en alumnos de este nivel.

¿Demuestra esto que la educación formal no enseña de por sí a configurar estrategias de aprendizaje? Solo podría responder para este grupo de alumnos y la respuesta es que en general, no se aprecia que los años de formación hayan tenido impacto en este aspecto. Solo los estudiantes número 6 , 8, 13,14 15 y 17 (uno de Ciencias Sociales, uno de Lengua y Literatura y cuatro de Matemática) ofrecen un perfil del que podría afirmarse que encaran la tarea con un enfoque profundo, verificándose que han desarrollado estrategias de aprendizaje.

Como ya lo mencioné en el análisis, estos resultados hacen pensar en la influencia del área de conocimiento predominante a la que se dedican en la configuración de estrategias. Existe la posibilidad de que esté "leyendo" más de lo que las entrevistas revelan: que mi conocimiento de los estudiantes -ya que han sido alumnos míos durante todo un año-, haya influido en la interpretación de las entrevistas, agregando más información de la que éstas brindan; los he visto desempeñarse, desde su ingreso en una gran variedad de situaciones y en diferentes asignaturas, debido al estrecho y continuo contacto que se mantiene con los alumnos en esta institución, sumándose esto a mi falta de experiencia en la investigación.

Considero necesario profundizar en las entrevistas, de modo de explorar más exhaustivamente la existencia o no de estrategias; analizar la toma de apuntes y la elaboración de esquemas y mapas conceptuales, contrastándolos con las fuentes de información para analizar la capacidad de reorganizar el conocimiento. Sería interesante realizar esta investigación en época de exámenes, cuando los alumnos disponen de todo el horario para poder planificar su estudio, junto al requerimiento de dominar los saberes. Es de suponer además que en ese momento cuentan con todo el material que pudieran consultar, además de la participación de los docentes en clases de apoyo.

Debería además ampliarse la población estudiada, ya que si bien representa un porcentaje cercano al 50\% de la totalidad de alumnos de esas áreas, por tratarse de procedimientos de índole psíquica, no accesibles directamente al estudio, en un grupo de mayor tamaño se podrían buscar ciertas regularidades, si es que éstas se producen. Una investigación en esta área podría ser el inicio de un proyecto de investigación pedagógica, que no ha sido frecuente hasta ahora en el nivel de formación docente en nuestro país, caracterizado por propuestas discontinuas y atomizadas. Se trataría de un esfuerzo tendiente a la búsqueda de información sobre un asunto concreto en un tiempo y un lugar dados, que serviría de base para el diseño de acciones específicas con el objetivo de lograr una mejora en la calidad de los aprendizajes. 


\section{Bibliografía}

BLANCHET, A., GHIGLIONE, R. y otros. 1989. Técnicas de investigación en Ciencias Sociales. Madrid, Narcea.

COLL, C. PALACIOS, J., MARCHESI, A.(Comp).1988. Desarrollo psicológico y educación II. Psicología de la Educación. Madrid: Alianza.

MARCHESI, A., MARTIN, E. 1998. Calidad de la enseñanza en tiempos de cambio. Madrid: Alianza. MONEREO, C. 1998. Estrategias de enseñanza y aprendizaje. Formación del profesorado y aplicación en la escuela. $5^{\text {a }}$ ed. Barcelona:Grao.

MONEREO, C. 1995. Estrategias para aprender a pensar bien. Rev. Cuadernos Pedagógicos $\mathrm{N}^{\circ} 235$, pp. 34-52.

PERKINS, D.1995. La escuela inteligente. Del adiestramiento de la memoria a la educación de la mente. Barcelona: Gedisa.

POZO, J. 1998. Aprendices y maestros. Madrid: Alianza.

POZO, J., MONEREO, C. 1999. El aprendizaje estratégico. Enseñar a aprender desde el currículo. Madrid: Santillana.

RESNICK, L., KLOPFER, L., (Comp.) 1996. Currículum y cognición. Buenos Aires: Aique.

SIERRA BRAVO, R. 1998. Técnicas de Investigación social. Teoría y ejercicios. Madrid: Paraninfo.

TAYLOR, S.J. y BOGDAN, R. 1987. Introducción a los métodos cualitativos de investigación. Buenos Aires: Paidós.

TORRES, M. 1996. Formación docente: clave de la reforma educativa en Nuevas formas de aprender y enseñar. Santiago de Chile: Unesco.

* Diploma en Educación, Universidad ORT Uruguay. 